\title{
Daytime Development of the Boundary Layer over a Plain and in a Valley under Fair Weather Conditions: A Comparison by Means of Idealized Numerical Simulations
}

\author{
STEFANO SERAFIN* AND DINO ZARDI \\ Atmospheric Physics Group, Department of Civil and Environmental Engineering, University of Trento, \\ Trento, Italy
}

(Manuscript received 28 July 2010, in final form 28 November 2010)

\begin{abstract}
The daytime thermal structures of the valley boundary layer (VBL) and of the convective boundary layer (CBL) above a plain, as revealed by idealized large-eddy simulations, are compared. Simulations in the two environments consider similar thermal forcing, thus allowing an analysis of the atmospheric heating processes in the VBL and CBL in light of the volume-effect theory, traditionally invoked to explain the larger diurnal temperature ranges observed in valleys. It is found that, after an equal input of thermal energy, the atmospheric volumes affected by thermal perturbations in the $\mathrm{CBL}$ and in the VBL are comparable. Although the boundary layer top is higher in the VBL than in the CBL, the average VBL depth is approximately equal to the CBL depth, since the ground elevation is nonuniform in the valley. Accordingly, the volume-averaged potential temperature increments in the CBL and VBL are comparable. Nevertheless, surface air temperature variations are larger in the VBL, while differences in the thermal structures of the CBL and the VBL are found to be larger at elevated levels. These effects are related to the heat and mass transfer processes associated with upslope flows and midvalley subsidence. As far as the simulated CBL and VBL cases are representative of two asymptotic regions (respectively, far up valley and far over the plain) of a plain-valley system with a horizontal floor, their comparison provides insight in the mechanisms responsible for the generation of the pressure contrasts driving a daytime plain-to-valley wind at lower levels and possibly a valley-to-plain upper flow.
\end{abstract}

\section{Introduction}

A diurnal regime of alternating up- and down-valley winds is known to occur in mountain areas under fair weather conditions: air generally flows from the mouth of valleys to their head during the daytime, and in the opposite direction during the night (Whiteman 2000; Zardi and Whiteman 2011). This breeze regime is originated by a daily periodic variation in the thermal contrast between the atmosphere within a valley and that above an adjacent plain, the diurnal temperature range being larger in valleys. The more pronounced daytime temperature increase observed in valleys is commonly explained on the basis of

\footnotetext{
* Current affiliation: Department of Meteorology and Geophysics, University of Vienna, Vienna, Austria.

Corresponding author address: Stefano Serafin, Department of Meteorology and Geophysics, University of Vienna, Althanstraße 14/UZAII/2G507, A-1090 Vienna, Austria.

E-mail: stefano.serafin@univie.ac.at
}

a "volume effect" theory (Wagner 1932; Steinacker 1984; Whiteman 1990): since the air volume, and hence the air mass, below a reference top surface is smaller in a valley than over a plain, an equal heat input causes larger warming there.

The impact of this predicted larger warming on the intensity of valley breezes was studied by Vergeiner (1987). Based on an evaluation of the parameter dependence of an analytical model, Vergeiner (1987) suggests that the breeze circulation is expected to have a weak intensity and display a large phase delay (up to $6 \mathrm{~h}$ with respect to the start of heating) in comparatively long, shallow, or narrow valleys, and conversely to approach the very fast response typical of slope winds in comparatively short, deep, or broad valleys.

A further conceptual bulk model of up-valley flow in valleys, both with and without tributaries, was formulated by Egger (1990). The results of that study do capture the main features of the flow field in valleys (e.g., up-valley propagation of a cool breeze front, presence of a subsiding stably stratified valley core, importance of 
topographic factors) and provide quantitatively reasonable estimates of the intensity of valley breezes, but they appear to be extremely sensitive to the treatment of heat exchange from the slope wind layer to the free atmosphere, eventually predicting outflow from the valley mouth (contrary to expectations) when all heat from the slope layers is released to higher levels.

More recent studies showed that, indeed, circulations originated by slope winds and possibly extending well beyond mountaintops are a key factor in explaining the thermal structure of the valley boundary layer (VBL). For instance, recently Serafin and Zardi (2010a) studied the heat transfer processes occurring in a stretch of an idealized, infinitely long valley with a horizontal floor and a constant cross section. In this case, when the thermal forcing is invariant along the valley, the dynamics are two-dimensional and invariant along the valley as well. They showed that, while the atmosphere is warmed by midvalley subsidence during the morning transition phase, as originally suggested by Whiteman and McKee (1982), the heating process in the afternoon is dominated by turbulent convection, mostly generated by buoyancy effects near the surface.

The interplay between the "top-down" advective warming induced by subsidence and the "bottom-up" turbulent warming produced by convection affects the development of the thermal structure of the VBL. In particular, vertical potential temperature profiles in valleys can display elevated weakly turbulent and slightly stable layers surmounting a shallow mixed layer (ML). The possible occurrence of multiple layering was also observed in the vicinity of an isolated slope by Reuten et al. (2007, laboratory experiments) and by Serafin and Zardi (2010b, numerical simulations). These peculiar dynamics cause the vertical thermal structure of the VBL to be remarkably different from that of a convective boundary layer (CBL) developing above flat terrain (see, e.g., de Franceschi et al. 2003; Rampanelli and Zardi 2004; Rotach and Zardi 2007).

The differing vertical structure of the atmosphere over the plain and far up valley is a significant contribution to the onset of the along-valley wind system, since horizontal thermal imbalances are responsible for the generation of horizontal pressure gradients. A further important contribution, common to many natural valleys, is related to the existence of a sloping valley bottom, which significantly enhances the longitudinal component of the breeze, exactly in the same way as sloping sidewalls favor the onset of anabatic winds. The latter effect vanishes in valley-plain systems with a perfectly horizontal valley floor and invariant valley cross section, but a valley breeze is observed to develop even in such cases (Vergeiner 1987; Egger 1990; Rampanelli et al. 2004; Schmidli and Rotunno 2010; Schmidli et al. 2011). Consequently, the daytime potential temperature profiles developing far from a valley inlet-up valley and above the plain, respectively-can be considered as two asymptotic situations whose contrast would lead to the onset of an along-valley wind.

Indeed, analyzing the onset of the breeze system in an idealized valley with horizontal floor and in an initially resting atmosphere, Rampanelli et al. (2004) observed that the valley breeze originates at the valley mouth, where strong horizontal thermal gradients occur between the VBL and the CBL, and then spreads both downstream along the valley and upstream on the plain as the diurnal heating cycle progresses. This means that, in regions sufficiently far from the mouth of a horizontal valley, a large fraction of the daytime phase is characterized by a negligible or totally absent valley breeze, and slope flows are the dominant process. Slope winds, occurring at small spatial scales and thus quickly reacting to surface forcing, are indeed a key factor in the buildup of the thermal contrasts that cause valley flows (Vergeiner and Dreiseitl 1987): it is not until a few hours from sunrise that the valley breeze becomes the dominant feature of the flow field far up valley.

In the present analysis we concentrate on the daytime formation of the valley-plain temperature contrasts producing the pressure imbalance to which the up-valley wind system responds. Accordingly, we adopt a simplified model design: the onset of the above-mentioned thermal contrast is studied by means of separate numerical simulations, representing, respectively, the prototypes of the CBL and VBL. To this purpose we exploit results reported in Serafin and Zardi (2010a). In particular, we compare the large-eddy simulations of the VBL described therein with a simulation representing the growth of the CBL in a plain region subject to identical radiative forcing. The numerical model in use is described in section 2 , while the CBL simulation is presented in section 3. In section 4 we recall the main features of the VBL dynamics outlined in Serafin and Zardi (2010a), evaluate the effects of the valley-cross-section geometry on the observed bulk heating of the VBL, and make comparisons between the thermal structures of the CBL and VBL. Conclusions are drawn in section 5 .

\section{Numerical simulations}

The simulations presented in this study have been carried out with the Advanced Regional Prediction System (ARPS) model (Xue et al. 2000, 2001), which includes prognostic equations for the three components of momentum, potential temperature, and pressure, as well as for the subgrid-scale turbulent kinetic energy. 
Three simulations of the boundary layer (BL) development were carried out. The first one (section 3) represents a prototype of the daytime evolution of the CBL above flat terrain under fair weather conditions - that is, undisturbed by any synoptic-scale forcing. Two more simulations (section 4) represent the evolution of the VBL in an equally unperturbed situation, in valleys with a triangular and a trapezoidal cross section, respectively. Both valleys are $2000 \mathrm{~m}$ deep and have $30^{\circ}$ slopes, while they differ in the floor width (0 and $5000 \mathrm{~m}$, respectively). Hereafter, these two simulations are referred to as the "narrow valley" and "wide valley." The horizontal dimensions of the three domains are of $5000 \mathrm{~m} \times 5000 \mathrm{~m}$ for the CBL simulation and of $5000 \mathrm{~m} \times 8400 \mathrm{~m}$ and $5000 \mathrm{~m} \times 13600 \mathrm{~m}$ for the two valley simulations, respectively. Because of the small horizontal length scales of the processes in exam, Coriolis effects are not considered in the present analysis.

The geometrical features of the two valley domains have been designed to represent the typical scales of Alpine valleys. Of course, this constant-cross-section valley geometry cannot allow us to appreciate the consequences of the onset and propagation of the valley breeze, which is one of the major features of the flow field in real valleys during the afternoon. Also, the assumption of negligible synoptic forcing, which implies the absence of an ambient wind above the valley crests, prevents any accounting for large-scale heat advection effects. Both of these phenomena can have a relevant impact on the dynamics of the CBL in mountainous regions. However, notice that on one hand thermally driven flows normally reach their maximum development exactly when the pressure field is leveled, and on the other hand valley winds react to thermal forcing much more slowly than slope flows. Consequently, even neglecting the above-mentioned forcings, the present numerical setup seems appropriate to characterize the buildup of valley-plain thermal contrasts, at least during the morning phase.

A stretched terrain-following computational mesh is used, with a minimum vertical grid spacing of $20 \mathrm{~m}$ at the surface and a resolution of $50 \mathrm{~m}$ in both horizontal directions. The ratio between the horizontal and vertical grid spacing is chosen so as to minimize the risk of inaccurately computing pressure gradients near sloping surfaces (De Wekker 2002), in the case of valley simulations. The depth of all three simulation domains is of $8000 \mathrm{~m}$ : this considerable vertical extent allows us, in the valley simulations, to accurately represent the thermal plumes that develop at the top of valley walls and overshoot into the free atmosphere up to a height of about $4000 \mathrm{~m}$. A 2000-m-deep Rayleigh damping layer is included below the domain top to absorb vertically propagating internal waves and prevent their spurious reflection. An initially resting dry atmosphere, linearly stratified with $\partial \theta / \partial z=3 \times 10^{-3} \mathrm{~K} \mathrm{~m}^{-1}$, is considered $(\theta$ denotes the potential temperature). Boundary conditions are periodic on all lateral boundaries, while both the domain top and the ground surface are considered impermeable.

Large-eddy simulations (LES) of the flow field in the atmospheric BL are carried out using the order-1.5 subgridscale flux model by Moeng and Wyngaard (1984). Simulation results are subject to an averaging operation in successive 10-min windows to approximate the ensemble mean properties of the flow field.

Land surface processes governing the soil-atmosphere energy exchange are parameterized with the Noilhan and Planton (1989) model, with radiative forcing evaluated from a simplified balance between the absorbed solar radiation and the absorbed and emitted longwave radiation. All simulations start at 0900 LST, when the surface sensible heat flux, as determined by the surface energy budget, becomes positive. This relatively late start of the diurnal heating phase depends on the combined effect of the initial conditions (a resting atmosphere with no shear turbulence production) and the peculiar soil properties (dry soil with low heat capacity). This point will be explained more extensively in section 3 below.

Results from the two valley simulations are extensively analyzed in Serafin and Zardi (2010a), where further details about the model setup can be found.

\section{CBL above homogeneous terrain}

Figure 1 shows the main features of the CBL growth above a plain. The surface heat flux trend observed in this simulation (negligible magnitude in the morning, followed by a sharp increase before noon) is rather particular, but it can be explained considering the important role of turbulent atmospheric convection in enhancing the heat transfer from the ground surface in a dry, desert-like environment (Oke 1987). Despite the strong radiative forcing $\left(1100 \mathrm{~W} \mathrm{~m}^{-2}\right.$ global solar radiation, $600 \mathrm{~W} \mathrm{~m}^{-2}$ net radiation), sensible heating grows initially very slowly, because most of the available radiant energy contributes to heating the ground. Meanwhile, very little thermal energy is transferred from the ground to the resting atmosphere in the absence of surface wind gusts. However, as soon as the soil temperature becomes high enough, turbulent convection is triggered, as the initial stability of the near-surface atmosphere is overcome. Then, surface wind gusts caused by turbulent eddies enhance the sensible heat flux, in a sort of positive feedback process.

The surface sensible heat flux peaks at approximately $0.4 \mathrm{~K} \mathrm{~m} \mathrm{~s}^{-1}\left(\approx 500 \mathrm{~W} \mathrm{~m}^{-2}\right.$ at hour 4$)$, while the surface momentum flux, quantified by $u_{*}$, is $0.25 \mathrm{~m} \mathrm{~s}^{-1}$ at most, between hours 4 and 6 . A comparison of these figures with those referred to the valley simulations in Serafin and Zardi (2010a) reveals that the surface thermal 

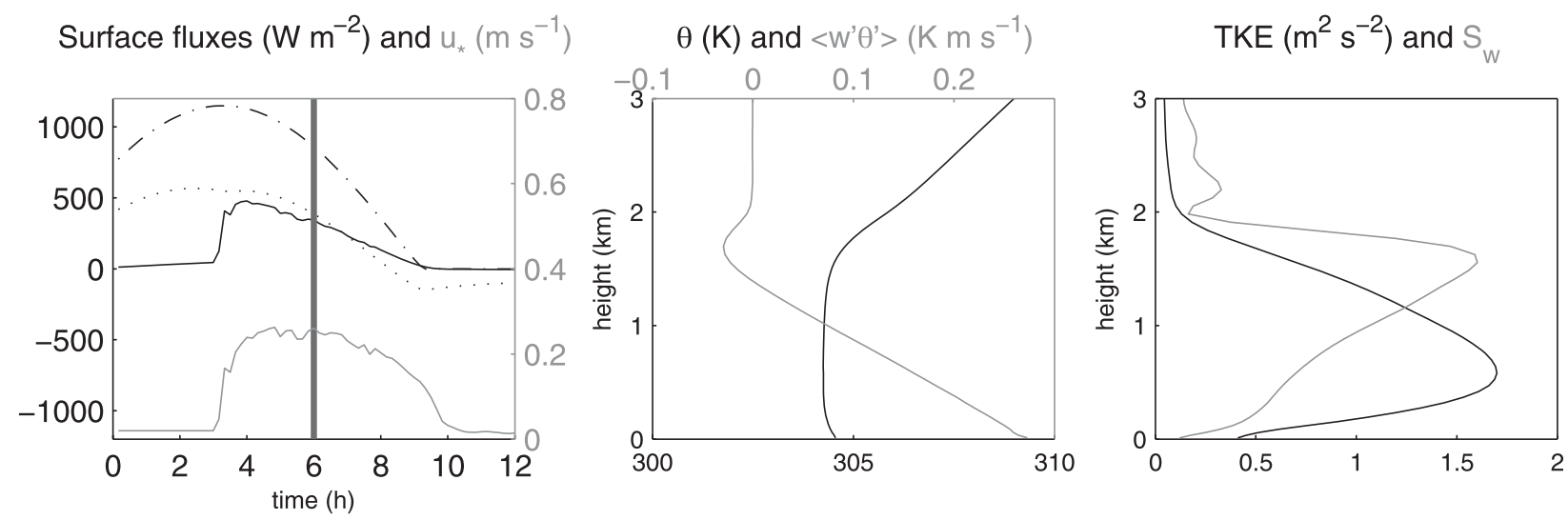

FIG. 1. Time evolution and vertical structure of characteristic quantities in the CBL. (left) Time evolution of the surface energy and momentum budget. [Left axis: shortwave radiation, net radiation, and sensible heat flux, plotted using dash-dotted, dotted, and continuous black lines, respectively. Hour 0 corresponds to 0900 LST. Right axis: friction velocity, plotted using a gray line. Bottom axis: time (h).] (middle) Vertical profiles of potential temperature (black line, bottom axis) and turbulent heat flux (gray line, top axis). (right) Turbulent kinetic energy (black line, bottom axis) and vertical velocity skewness (gray line, top axis).

forcing on the valley atmosphere is not significantly different from that on the plain, given an equal radiant energy input. In contrast, momentum fluxes appear to be greatly enhanced over the slopes in comparison to horizontal terrain.

After $6 \mathrm{~h}$ of runtime, the CBL above flat terrain displays a 1900-m-deep ML, surmounted by an 800-m-deep entrainment layer (EL). The ML is characterized by a linearly decreasing vertical heat flux profile, with an entrainment-to-surface heat flux ratio of around 0.15 as expected (see, e.g., Sullivan et al. 1998). Coherently, potential temperature is almost constant across the ML.

The turbulent kinetic energy peaks at $1.8 \mathrm{~m}^{2} \mathrm{~s}^{-2}$ in the lower third of the CBL depth, while the vertical velocity skewness $\left(S_{w}=\overline{w^{\prime 3}} / \overline{w^{\prime 2}} 3 / 2\right.$ is positive throughout the ML, indicating that thermal updrafts are stronger and narrower than downdrafts.
This picture agrees qualitatively well with textbook concepts about the growth of a CBL above flat terrain (see, e.g., Stull 1988).

The left panel in Fig. 2 shows the time evolution of the thermal structure of the CBL, by means of a time-height cross section: here, regions of warming and cooling over time are identified, respectively, by the upward or downward shift of isentropes with respect to the initial condition. For instance, a continuous downward bending of isentropes indicates progressive warming of the atmosphere. The CBL growth is maintained by turbulent convection, which transports heat upward from the ground surface (where $\partial \theta / \partial z<0$ ). The ML develops starting approximately at hour 3 of the simulation. Thermals overshooting above their level of neutral buoyancy cause turbulent entrainment, and mix air from the EL downward to the bulk of the ML. This results in
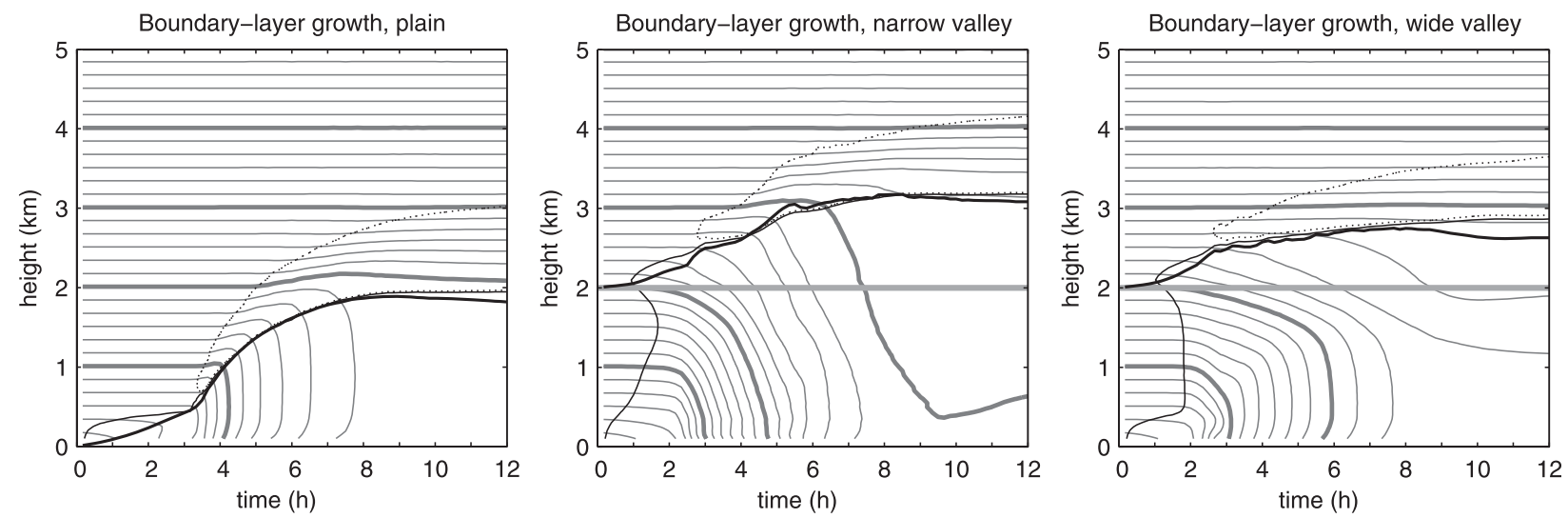

FIG. 2. Time-height series of mean potential temperature in the CBL. Isolines every $0.5 \mathrm{~K}$, thick every $3 \mathrm{~K}$. The thin continuous and dotted black lines mark regions with, respectively, positive and negative deviations from the unperturbed initial profile. The thick black line indicates an estimate of the CBL depth according to Eq. (1). The horizontal gray line indicates the sidewall top level. 
potential cooling in the EL above the ML, and in the formation of a strong capping inversion.

The neutral buoyancy level of parcels heated at the surface can be estimated as

$$
z_{\mathrm{lnb}}=\frac{\theta_{\mathrm{sfc}}-\theta_{\mathrm{sfc}}^{0}}{\Gamma}
$$

where $\theta_{\text {sfc }}$ is the instantaneous surface potential temperature, $\theta_{\mathrm{sfc}}^{0}$ the initial value of the same parameter, and $\Gamma=3 \times 10^{-3} \mathrm{~K} \mathrm{~m}^{-1}$ quantifies the background thermal stratification.

Note that $z_{\operatorname{lnb}}$ is a good predictor of the ML depth, as shown by the thick black line in Fig. 2: $z_{\operatorname{lnb}}$ increases in time, in exact coincidence with the zero turbulent heat flux height. This is an obvious finding, considering that the growth of this shear-free CBL is entirely governed by the buoyancy generated by surface heating.

\section{CBL and VBL: A comparison}

A distinctive feature of the VBL, in comparison to the rather simple situation of the CBL outlined in section 3 , is the development of persistent and organized surface winds along the slopes. These induce a compensating sinking motion at the valley core, producing a considerable impact on the vertical structure of the BL. Differences between the bulk properties of the CBL and the VBL are outlined in detail below.

\section{a. BL growth mechanisms}

Figure 2 is exactly analogous to Fig. 8 in Serafin and Zardi (2010a), partially reproduced here for the reader's convenience. A comparison of the three simulations reveals that, both in the plain and in the mountain case, the growth of the BL (i.e., in this context, the atmospheric layer thermally perturbed by diurnal circulations) is controlled by the vertical extent of thermal plumes. However, in the CBL thermals are free to develop anywhere on the ground surface, whereas in the VBL they are anchored to elevated mountaintops. Owing to this high-altitude heat input, the VBL displays a considerably larger vertical extent than the CBL.

Serafin and Zardi (2010a) showed that nonturbulent advection is an important process in redistributing heat in the VBL. This can be appreciated from the timeheight cross sections in Fig. 2, considering the shape of isentropes below the neutral buoyancy level. The latter are approximately vertical above the plain, showing that a well-mixed atmospheric layer is subject to the same heating rate at all levels. On the contrary they are slanted in valleys (at least at an early stage and immediately below crest height), suggesting that a stable atmospheric layer is being displaced downward, favoring the descent of potentially warmer air. This observation supports the concept that the top-down advective warming produced by midvalley subsidence is a key process in the heating of a valley, in addition to the bottom-up uniform turbulent heating producing a growing ML.

Therefore, unlike what happens in the CBL, a large amount of the surface heat input is transferred by nonturbulent advective motions in the VBL. Serafin and Zardi (2010a) showed that, indeed, these motions damp convective turbulence in a valley, at least initially.

One further remarkable difference between the CBL and VBL is that downward turbulent heat fluxes at the BL top (entrainment fluxes) can be considerably stronger in the CBL, as can be judged by the larger upward shifting of isentropes above the LNB in that case (Fig. 2): the footprint of turbulent entrainment (negative $\theta$ perturbations with respect to the linearly stratified initial state) is well visible above the plain and in the narrow valley, while it almost vanishes in the large valley.

A possible explanation for this phenomenon is that, compared to what happens in the CBL, thermal updrafts in the VBL are distant and separated by larger downdraft regions, especially if a wide valley floor exists between ridges. Increased spacing between thermals, due to the orographic control of their "roots," seems to cause a significant reduction of the overall effects of entrainment fluxes.

\section{b. Surface temperature tendency}

In Fig. 3 the tendencies of the mean surface temperature in the three simulations are compared. For the valley cases, we consider the valley floor temperature.

Compared to what happens above the plain, the sensible heat and momentum fluxes start increasing about $1 \mathrm{~h}$ earlier in the two valley runs (after $2 \mathrm{~h}$ of simulation time, rather than $3 \mathrm{~h}$ ). Indeed surface fluxes are heavily controlled by the surface wind fields, and the onset of upslope flow occurs faster than turbulent convection, resulting in an earlier heating of the atmosphere in the valley than above the plain. This timing might not be observed in a real valley, where shading effects would be crucial in delaying the local sunrise time and the initial stage of the morning transition phase.

The steepness of the surface temperature tendency is larger in the valley simulations (Fig. 3, left). Furthermore, in the valley atmosphere subsidence warming appears to be stronger than turbulent convection warming. This is more easily appreciated from an inspection of the surface temperature tendency (Fig. 3, right). Here $\left.(\partial \theta / \partial t)\right|_{\text {sfc }}$ appears to have two maxima in each of the valley simulations, and only one in the flat model run. Peaks occur, respectively, at hours 2 and 5 in the narrow valley, at hours 2 and 3 in the wide valley, and at hour 3 in the flat domain. 

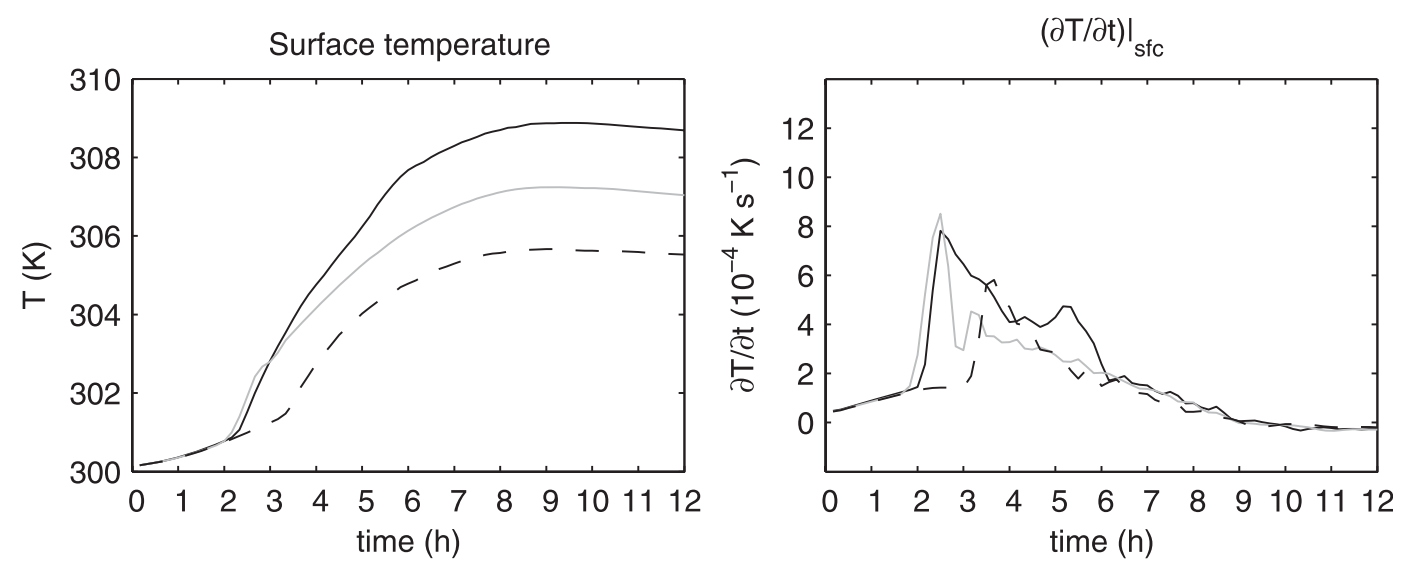

FIG. 3. Evolution of (left) the surface temperature and (right) the surface temperature tendency in time. Continuous black line: narrow valley floor. Gray line: wide valley floor. Dashed black line: plain.

The first maximum in the surface temperature tendency in the valley simulations is related to subsidence warming $(-w \partial \theta / \partial z)$ : the peak value of $8 \times 10^{-4} \mathrm{~K} \mathrm{~s}^{-1}$ is compatible with a downward vertical wind speed of about $0.25 \mathrm{~m} \mathrm{~s}^{-1}$ and a thermal stratification of $3 \times 10^{-3} \mathrm{~K} \mathrm{~m}^{-1}$, as observed in the simulations by Serafin and Zardi (2010a). The peak occurs in both cases at hour 2, exactly when the alongslope anabatic wind starts blowing, triggering compensating subsidence in the valley core.

The second peak in the surface temperature tendency is instead related to the onset of turbulent convection: surface wind gusts, caused by large turbulent eddies, enhance the heat transfer from the ground to the atmosphere. Both in the narrow and in the wide valley, the second peak occurs precisely when upslope flows weaken, as an effect of the increased turbulent mixing (Fig. 2 in Serafin and Zardi 2010a). This occurs later in the narrow valley, and therefore the warming effect of subsidence is maintained for longer there.

In the flat terrain simulation, the surface temperature tendency peaks between hours 3 and 4, again in connection with the onset of turbulent convection. After the initial peak, the growth rate of potential temperature decreases, as a consequence both of the decreasing sensible heat flux and of the increasing turbulent mixing, which transports heat from the vicinity of the surface toward higher altitudes.

To summarize, Fig. 3 suggests that the subsidence of potentially warm air is a more organized and efficient heating mechanism than turbulent convection and competes with it. The former does not occur above homogeneous terrain, but only over topographic structures. There, it can be effective for a longer period if the valley geometry is more favorable: the narrower the valley, the stronger will be the midvalley subsidence, damping turbulent convection and delaying its onset and thus allowing the slope flow to persist for a longer time without being affected by the onset of large turbulent eddies.

This possibly explains why surface temperature increases at a stronger rate during daytime in valleys than in flat regions. However, it is not implied that surface temperature maxima will invariably be higher in valleys than above adjacent plains, since the local sunrise and sunset time, the possibly different sensible heat flux in the two regions, and the initial valley-plain surface temperature contrast may also play a role.

\section{c. Geometric effects on the bulk heating of a valley: Theory}

An immediate consequence of the analysis provided by Serafin and Zardi (2010a) is that the thermal structure of the VBL is not, in general, horizontally homogeneous. For instance, vertical potential temperature profiles are expected to be significantly different between the valley core region (where subsidence occurs and convective turbulence is damped) and the mountaintops (where vigorous thermal updrafts develop, causing significant entrainment of air from the EL).

Anyway, if enough time is allowed, even the VBL becomes fairly well mixed, and horizontal thermal inhomogeneities between the valley core and the slopes will be reduced. Serafin and Zardi (2010a) showed that, although a given isentropic surface will move up or down throughout the daily cycle depending on local cooling or heating, its mean profile will not deviate appreciably from the horizontal, except very near the mountaintops.

Therefore, if sufficiently long time periods are considered (e.g., intervals of a few hours), the VBL can be appropriately characterized by means of the vertical profiles and bulk values of its properties. This proves useful for understanding how the geometry of a valley 


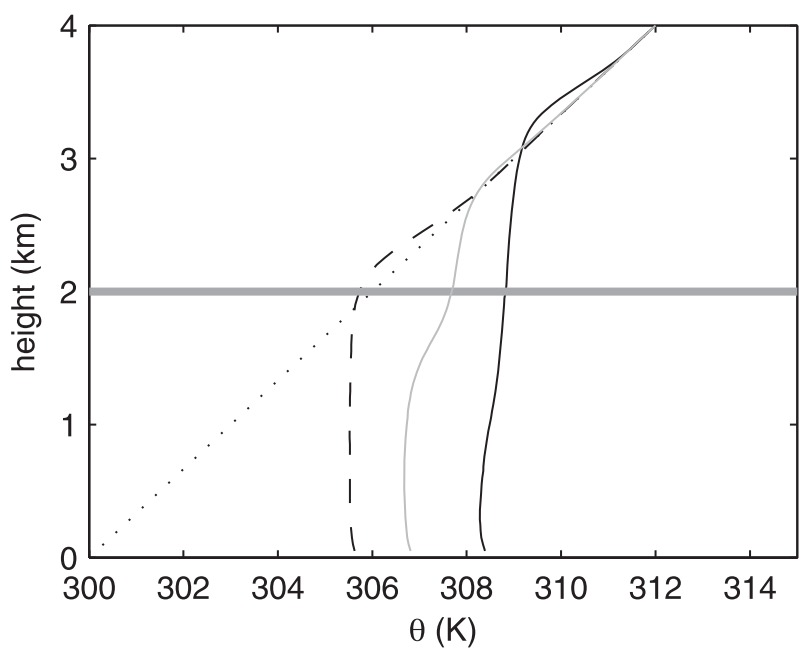

FIG. 4. Vertical potential temperature profiles in the flat (dashed black line, referred to $t=29400 \mathrm{~s}$ ), wide valley (gray line, $t=$ $25200 \mathrm{~s}$ ), and narrow valley (continuous black line, $t=25200 \mathrm{~s}$ ) simulation domains. The different times correspond to an equal energy input for all cases: see section $4 \mathrm{~d}$ for explanations. The horizontal gray line indicates the valley sidewall top level.

cross section affects the overall heating of the valley atmosphere, as shown below.

Figure 4 compares the mean vertical potential temperature profiles observed in the three domains in exam (plain, narrow, and wide valley) after an equal energy input (see section 4d). Horizontal averaging was performed to obtain the profiles, in two dimensions in the CBL simulations and only along the valley axis in the VBL simulations. The two valley profiles have slightly different features: while the VBL is almost perfectly neutral in the narrow valley, it is characterized by two well-mixed regions separated by a weakly stable layer in the wide one. Strong upslope flow and subsidence in the narrow valley cause the VBL to become well mixed. Weaker advective warming in the wide valley and stronger turbulent convection at its floor cause instead the observed layering there. Anyway, since the observed potential temperature variations do not exceed $1 \mathrm{~K}$ through a 2-km layer, we can consider the VBL fairly well mixed in this case too.

Figure 4 shows that the atmosphere in valleys is characterized by a larger $\theta$ increase compared to the plain: the narrower the valley, the larger the valley-plain $\theta$ difference throughout the BL depth. Furthermore, the higher temperature is related to a larger BL depth: the VBL of both valleys is deeper and warmer than the CBL over the plain area.

This can be explained by integrating the heat equation in space and in time, considering the role of the valley geometry. The first law of thermodynamics at any point in the atmosphere can be written

$$
\rho c_{p} \frac{T}{\theta} \frac{d \theta}{d t}=-\frac{\partial Q_{i}}{\partial x_{i}}
$$

where $Q_{i}$ are the components of the diabatic heat flux, positive outward (Gill 1982). Using the mass continuity constraint, Eq. (2) can be elaborated to obtain

$$
\frac{\partial \rho \theta}{\partial t}+\frac{\partial \rho \theta u_{i}}{\partial x_{i}}=-\frac{1}{c_{p}} \frac{\theta}{T} \frac{\partial Q_{i}}{\partial x_{i}} .
$$

After Reynolds decomposition $\left(\rho=\bar{\rho}+\rho^{\prime}, \theta=\bar{\theta}+\theta^{\prime}\right.$, $\left.u_{i}=\bar{u}_{i}+u_{i}^{\prime}\right)$ and averaging, and upon use of the Boussinesq approximation to eliminate $\rho^{\prime}$, Eq. (3) becomes

$$
\frac{\partial \bar{\rho} \bar{\theta}}{\partial t}=-\frac{\partial \bar{\rho} \bar{\theta} \bar{u}_{i}}{\partial x_{i}}-\frac{\partial \bar{\rho} \overline{u_{i}^{\prime} \theta^{\prime}}}{\partial x_{i}}-\frac{1}{c_{p}} \frac{\theta}{T} \frac{\partial Q_{i}}{\partial x_{i}}
$$

or, denoting the components of the turbulent heat flux as $H_{i}=\bar{\rho} c_{p} \overline{u_{i}^{\prime} \theta^{\prime}}$ (positive outward, like $Q$ ),

$$
\frac{\partial \bar{\rho} \bar{\theta}}{\partial t}=-\frac{\partial \bar{\rho} \bar{\theta} \bar{u}_{i}}{\partial x_{i}}-\frac{1}{c_{p}} \frac{\partial H_{i}}{\partial x_{i}}-\frac{1}{c_{p}} \frac{\theta}{T} \frac{\partial Q_{i}}{\partial x_{i}} .
$$

According to Eq. (5), the heating of a parcel in the atmosphere results from the convergence of the advective, turbulent, and diabatic heat fluxes, as represented by the three terms on its rhs. Useful insight on the heating of the atmosphere within and above a valley can be obtained from an integration of Eq. (5) over a fixed volume of atmosphere $V$, invariant in time between two instants $t_{1}$ and $t_{2}$. Applying the divergence theorem,

$$
\begin{aligned}
\frac{\partial}{\partial t} \int_{V} \bar{\rho} \bar{\theta} d V= & -\int_{\partial V} \bar{\rho} \bar{\theta} \bar{u}_{i} n_{i} d(\partial V)-\frac{1}{c_{p}} \int_{\partial V} H_{i} n_{i} d(\partial V) \\
& -\frac{1}{c_{p}} \int_{V} \frac{\theta}{T} \frac{\partial Q_{i}}{\partial x_{i}} d V
\end{aligned}
$$

where $n_{i}$ denotes the components of the outward unit vector normal to the volume boundary $\partial V$. Equation (6) can become considerably simpler when applied to the results of our simulations, provided some assumptions are made. In particular,

- we include surface heating in the turbulent heat flux (second term on the rhs), as usually assumed in imposing the surface boundary conditions to numerical models. Hence, the third term on the rhs of Eq. (5) (mostly related to the radiative flux divergence, not accounted for in our simulations) can be considered negligible.

- The control volume $V$ shall be chosen so as to match the region of atmosphere where the thermal perturbation induced by the slope flow circulation between times $t_{1}$ and $t_{2}$ is relevant. Given the discussion at the 
beginning of the present section, the upper limit of the VBL will be taken as a horizontal surface immediately above the entrainment layer, at an altitude we denote by $h$. Thermal perturbations are actually negligible above $h$, as shown in Serafin and Zardi (2010a) and in section 3 of the present paper. Thus defined, $V$ incorporates both the ML (with positive $\theta$ perturbations) and the overlying EL (with negative $\theta$ perturbations). Consequently, the entrainment heat flux will not be included among the turbulent fluxes toward the volume $V$, since it merely redistributes thermal energy within it. This choice of $V$ is different from the approach adopted by Schmidli and Rotunno (2010), where the top surface of the valley control volume was a horizontal plane at the sidewall top level.

- Vertical fluxes related to the mean flow through the upper boundary of $V$ will approach zero, meaning that the total air mass within $V$ will be conserved at a very good approximation. Therefore the mean air density will remain approximately constant: we verified that $\bar{\rho}$ is subject to small variations in the VBL throughout our simulations (less than $1 \%$ on average). Note that we are not asserting that density variations are unimportant in themselves (in fact, they are a key factor in explaining the dynamics of slope and valley winds; see, e.g., Wenger 1923). We only suggest that their effect on the heat budget of the VBL is small enough to be neglected.

- The integrated horizontal fluxes, both turbulent and advective, through the lateral boundaries vanish in our simulations as a consequence of the periodic lateral boundary conditions: perturbations advected or diffused through a lateral boundary will reenter the simulation domain from the opposite side.

To summarize, in the idealized domains considered in the present simulations, we can appropriately assume that

- the diabatic heat flux divergence can be neglected,

- the turbulent flux at the ground surface is the only heat flux into volume $V$, and

- density at any point does not change significantly in time.

Consistently with the above assumptions Eq. (6) simplifies to

$$
\begin{aligned}
\int_{V} \bar{\theta}_{t_{2}} d V-\int_{V} \bar{\theta}_{t_{1}} d V & =-\frac{1}{\bar{\rho} c_{p}} \int_{t_{1}}^{t_{2}}\left[\int_{\partial V} H_{i} n_{i} d(\partial V)\right] d t \\
& =\frac{1}{\bar{\rho} c_{p}} \int_{t_{1}}^{t_{2}}\left(\int_{S_{0}} H_{0 i} n_{i} d A_{0}\right) d t
\end{aligned}
$$

where $H_{0 i}$ denotes the components of the surface sensible heat flux (positive inward, toward the atmosphere through the topographic surface of area $S_{0}$ ).
Now, $V$ may be expressed as $V=S(h-\bar{z})$, where $\bar{z}$ is the mean altitude of the ground surface above the valley bottom and $S$ is the projection of $S_{0}$ on the horizontal ( $\bar{z}=0$ and $S=S_{0}$ if the topography is flat). The mean sensible heat flux $\bar{H}_{0}$, and the final $\Theta_{t_{2}}$ and initial $\Theta_{t_{1}}$ mean potential temperatures of the $\mathrm{BL}$, can be defined, respectively, as

$$
\begin{aligned}
\frac{1}{S \Delta t} \int_{t_{1}}^{t_{2}}\left(\int_{S_{0}} H_{0 i} n_{i} d A_{0}\right) d t & \equiv \bar{H}_{0}, \\
\frac{1}{V} \int_{V} \bar{\theta}_{t_{2}} d V & \equiv \Theta_{t_{2}}, \\
\frac{1}{V} \int_{V} \bar{\theta}_{t_{1}} d V & \equiv \Theta_{t_{1}} .
\end{aligned}
$$

Definitions (9)-(11) can be substituted in Eq. (8), leading to

$$
\bar{H}_{0}=\bar{\rho} c_{p} \frac{(h-\bar{z})\left(\Theta_{t_{2}}-\Theta_{t_{1}}\right)}{\Delta t} .
$$

Equation (12) relates the mean potential temperature increment for an atmospheric layer below a height $h$ and over a time interval $\Delta t$ to the total inward heat flux and to the mean depth of the layer. Note that Eq. (12) descends from an approximate integration of an approximation of the heat equation; therefore it may be regarded as a diagnostic relationship with a margin of error of a few percent.

The derivation of Eq. (12) is in some aspects analogous to the procedure that leads to the definition of the area-height distribution concept (AHD; Steinacker 1984). Different hypotheses are made here about the control volume to be considered. Topography is also represented differently in the present analysis - that is, by means of its mean elevation rather than by its AHD.

Equation (12) can be used to evaluate the impact of the geometry of a valley on the heating rate of the atmosphere by considering a posteriori (i.e., diagnosed from simulations) estimates of its parameters, as shown in section $4 \mathrm{~d}$. A comparison of the potential temperature increase in different cases can be made after making sure that an equal energy input $\bar{H}_{0} \Delta t$ was provided to the atmosphere in all of them.

Note that in the ARPS model only the vertical component of the surface heat flux is imposed. In this particular case, since at any point on the surface $H_{0 i} n_{i}=H_{0} \cos \alpha$ and $d A_{0}=d A / \cos \alpha$ (where $\alpha$ is the slope angle), the definition of $\bar{H}_{0}$ can be further simplified to 
TABLE 1. Bulk properties of the boundary layer in the three simulations. See section $4 \mathrm{~d}$ for a detailed explanation.

\begin{tabular}{cllccc}
\hline \hline & Parameter & \multicolumn{1}{c}{ Units } & Plain & $\begin{array}{c}\text { Narrow } \\
\text { valley }\end{array}$ & $\begin{array}{c}\text { Wide } \\
\text { valley }\end{array}$ \\
\hline 1 & $\Delta t$ & $\mathrm{~s}$ & 29400 & 25200 & 25200 \\
2 & $\int_{0}^{\Delta t} H_{0} d t$ & $10^{6} \mathrm{~J} \mathrm{~m}^{-2}$ & 6.27 & 6.30 & 6.26 \\
3 & $h$ & $\mathrm{~m}$ & 2700 & 3800 & 3300 \\
4 & $\bar{z}$ & $\mathrm{~m}$ & 0 & 1000 & 580 \\
5 & $V=S(h-\bar{z})$ & $\mathrm{m}^{3}$ & $2700 S$ & $2800 S$ & $2720 S$ \\
6 & $\Theta_{t_{2}}$ & $\mathrm{~K}$ & 306.0 & 309.1 & 307.8 \\
7 & $\Theta_{t_{1}}$ & $\mathrm{~K}$ & 304.0 & 307.2 & 305.8 \\
8 & $\Theta_{t_{2}}-\Theta_{t_{1}}$ & $\mathrm{~K}$ & 2.0 & 1.9 & 2.0 \\
9 & $\theta_{t_{2}}^{\text {sfc }}-\theta_{t_{1}}^{\text {sc }}$ & $\mathrm{K}$ & 5.6 & 8.3 & 6.9 \\
10 & $\bar{H}_{0}$ & $\mathrm{~W} \mathrm{~m}{ }^{-2}$ & 214 & 246 & 251 \\
\hline
\end{tabular}

$$
\bar{H}_{0}=\frac{1}{S \Delta t} \int_{t_{1}}^{t_{2}}\left(\int_{S} H_{0} d A\right) d t
$$

\section{d. Geometric effects on the bulk heating of a valley: Verification}

As shown in section $4 \mathrm{~b}$, in our simulations the atmosphere above the plain feels an appreciable sensible heat flux later than the valley atmosphere. Hence, as the two environments are subject to approximately equal heat fluxes, a longer time is required for the same integral energy input to be provided to the valley atmosphere. For instance, it takes a little more than $8 \mathrm{~h}$ above the plain to achieve the same total energy input that is provided to the valley atmosphere in $7 \mathrm{~h}$ (lines 1 and 2 in Table 1: differences in $\int_{0}^{\Delta t} H_{0} d t$ amount to less than $\left.1 \%\right)$. This motivates the choice to consider the state of the atmosphere at $\Delta t=$ $29400 \mathrm{~s}$ above the plain and at $\Delta t=25200 \mathrm{~s}$ above the two valleys. Comparing the three simulations after an equal integral energy input has been imparted to the system, as suggested above, also eliminates any impact of a possible different timing of the surface heat flux: a different tendency of the (instantaneous) heat flux $H_{0}$ would only imply the selection of a different $\Delta t$, without further affecting the analysis.

The height $h$ right above the entrainment layer (line 3 in Table 1) can be evaluated directly from Fig. 4, from the separation of the instantaneous thermal profiles from those representing the unperturbed condition; it can also be estimated from Fig. 2, by considering the position of the upper limit of negative $\theta$ perturbations at the relevant time.

The height $\bar{z}$ (line 4 ) is calculated exactly based on the domain topography; $\Theta_{t_{2}}$ (line 6) and $\Theta_{t_{1}}$ (line 7) can be calculated exactly from the model output.

Other parameters in Table 1 are computed from those listed above or derived similarly from the model output. These include the total perturbed volume $V$ (line 5), the mean potential temperature increment in the BL (line 8 ), and the surface temperature increment (line 9).

Finally, Table 1 lists the mean heat flux into the $\operatorname{BL}\left(\bar{H}_{0}\right)$ diagnosed by means of Eq. (13) from the above-mentioned estimates of $h, \bar{z}, \Theta_{t_{2}}$, and $\Theta_{t_{1}}$. Estimates of $\bar{H}_{0}$ are in reasonable agreement with figures in lines 1 and 2 in Table 1. As expected, $\bar{H}_{0}$ is smaller above the plain, since it takes a longer time for the same thermal energy amount to be transferred to the atmosphere. The product $\bar{H}_{0} \Delta t$ is comparable in the three cases (variations are less than $3 \%$ ), which is reasonable since the total energy input is approximately the same. Finally, $\bar{H}_{0} \Delta t$ is in good agreement with $\int_{0}^{\Delta t} H_{0} d t$ (also within 3\%), meaning that the approximate integration that yields Eq. (12) is reasonably accurate.

Note that the overall volume of the VBL is comparable to that of the CBL (Table 1, line 5), unlike what is traditionally assumed by the volume-effect theory (e.g., Steinacker 1984). Since the heated volume above a valley and above a plain are of comparable size, the observed mean temperature increments are also comparable (Table 1, line 8: differences between the three simulations amount to less than $0.1 \mathrm{~K}$ ).

Nevertheless, it is still found that the surface potential temperature at the valley floor is subject to much larger increments ( $3 \mathrm{~K}$ more) than above the plain. This can be understood by considering that part of the heat input in a valley is provided to the atmosphere at a high altitude, up to the sidewalls top level. Hence, the VBL spans a larger vertical extent than the CBL and mixes potentially warmer air from high altitudes to the surface.

In other words, the depth of VBL during the day is always larger than the depth of the CBL because of the heat input occurring at mountaintops. Consequently, given the thermal stratification of the atmosphere, the mean potential temperature of this thick layer is initially warmer. During the daily phase, the VBL and CBL are subject to comparable mean potential temperature increments, and therefore the VBL remains on average potentially warmer than the CBL throughout the day. Moreover, both environments tend to become well mixed: as a consequence, in both cases the largest $\theta$ increments occur near the surface, since the atmosphere was initially stratified. The deeper the mixed layer, the (potentially) warmer the air mixed from aloft, the larger the surface temperature increment. Thus, for instance, the surface temperature in our simulations increases by up to $8.3 \mathrm{~K}$ in valleys and only by $5.6 \mathrm{~K}$ above the plain (Table 1, line 9).

The above discussion is clarified by Fig. 5, where a sketch of the bulk representation of the three BLs in exam (at the times reported in Table 1) is shown. A zeroorder approximation of the vertical potential temperature profiles, where $\theta$ equals $\Theta_{t_{2}}$ everywhere within the BL, is 

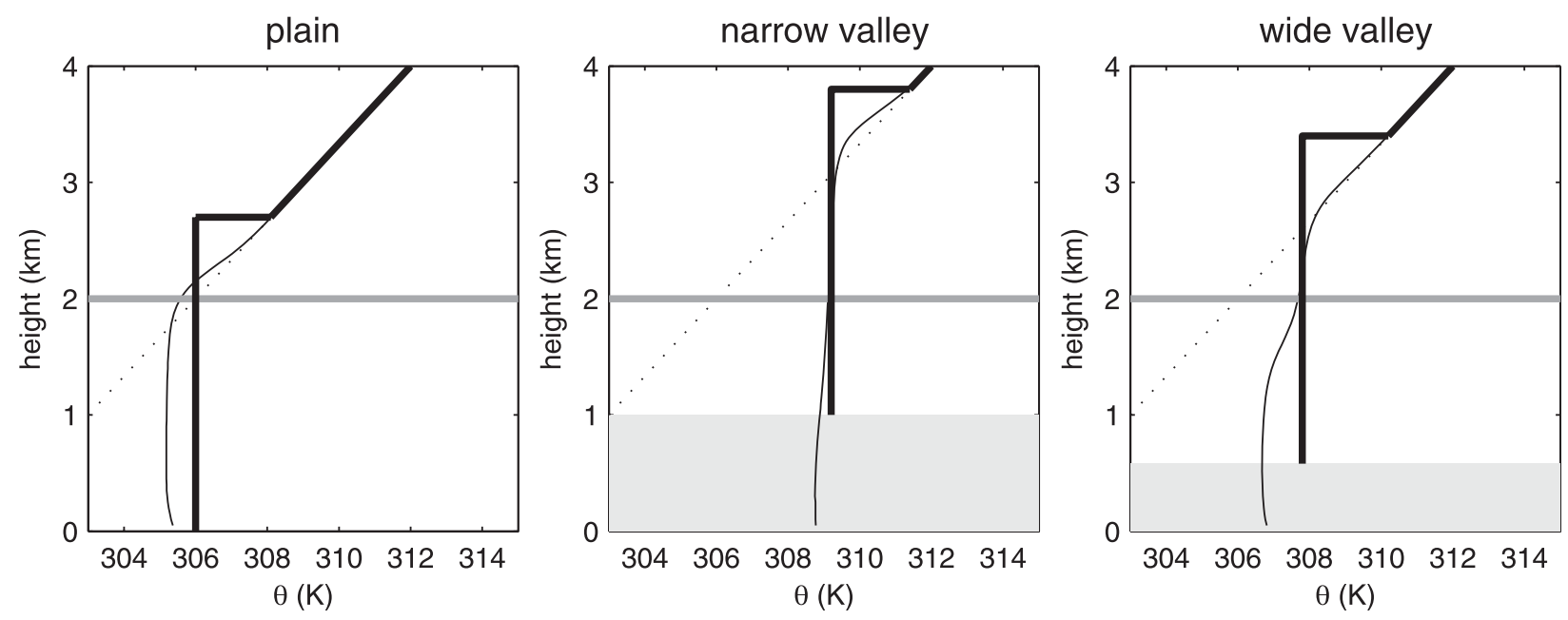

FIG. 5. Bulk representation of the (left) CBL and (middle),(right) VBLs in exam. Thin black line: cross-valley mean vertical $\theta$ profile. Thick black line: zero-order approximation of the $\theta$ profile. Dotted line: initial state. Gray shading: mean surface altitude $\bar{z}$. Horizontal dark gray line: valley sidewall top level.

also provided. Figure 5 shows that, if degraded to their zero-order approximations, the two VBLs and the CBL have comparable mean depth and are topped by an equally strong inversion. Compared to the CBL, the VBL extends to higher altitudes (and is consequently warmer on average) because it is subject to warming from a more elevated surface.

\section{e. Valley-plain temperature and pressure differences}

The vertical profiles of the valley-plain temperature and pressure differences are shown in Fig. 6. The largest temperature difference between the two regions appears to occur around the ridge-top level, where warming is produced by mountaintop plumes.

Temperature differences decrease rapidly above $1900 \mathrm{~m}$, at a rate of about $3 \times 10^{-3} \mathrm{~K} \mathrm{~m}^{-1}$. In fact, the depth of the ML over the plain is $1900 \mathrm{~m}$; above this height, temperature decreases at the adiabatic lapse rate of $9.8 \times$ $10^{-3} \mathrm{~K} \mathrm{~m}^{-1}$ in the VBL, while it decreases at lower rates above the CBL, where the atmosphere is stably stratified.

In the narrow valley, temperature differences become even negative higher up (Fig. 6). This happens because the VBL, which grows well higher than the CBL, is weakly entraining in this case. In the wide valley, where no evidence of entrainment is found (as discussed in section 4a), no negative valley-plain temperature differences occur. Below mountaintops, temperature differences remain positive, although they become smaller; also, they are smaller in the wide valley than in the narrow.

Although temperature differences between the valley and the plain are more relevant at elevated levels, the pressure difference is strongest near the surface. This can be explained assuming that the surface pressure depends on the total mass of the entire air column above, which is appropriate if the atmospheric motions preserve hydrostatic balance, as found in thermally driven along-valley flows (see Rampanelli et al. 2004; Schmidli and Rotunno 2010).

Accordingly, at any height $z$, the pressure difference between the valley and plain atmospheres can be related to the thermal contrast at higher levels, by vertically integrating the hydrostatic balance equation in a layer between $z$ and the BL depth $h$. As the thermal structure of the valley and plain atmospheres is unperturbed above $h$, it can be demonstrated that

$p_{\text {valley }}^{\kappa}(z)-p_{\text {plain }}^{\kappa}(z)=\frac{g p_{0}^{\kappa}}{c_{p}} \int_{z}^{h}\left(\frac{1}{\theta_{\text {plain }}}-\frac{1}{\theta_{\text {valley }}}\right) d z$,

where $p_{0}=1000 \mathrm{hPa}$. Figure 7 shows that the diagnostic relationship provided by Eq. (14) is rather accurate: at all levels, the estimated and the observed pressure differences match closely. A similar approach was used by Rampanelli et al. (2004) to estimate surface pressure gradients from horizontal thermal gradients aloft.

Based on the above discussion we conclude that, generally, surface pressure differences between valleys and adjacent plains can even be created by thermal imbalances occurring only at higher levels. In a region where the plain and valley environments were adjacent to each other (like those studied by Rampanelli et al. 2004; Schmidli and Rotunno 2010) a negative valleyplain pressure difference could develop even if the surface temperature were lower within the valley, provided 

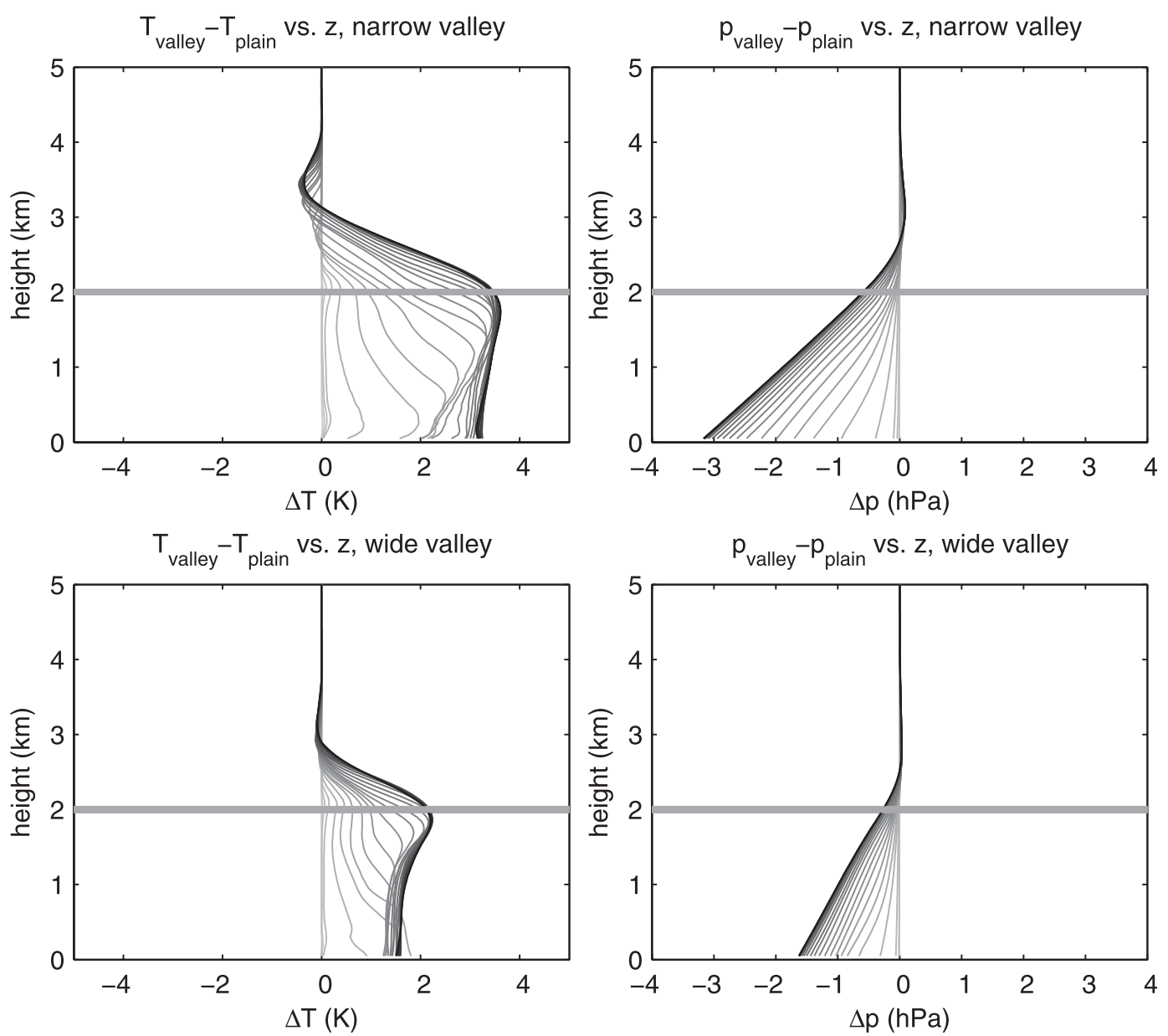

FIG. 6. Vertical profiles of temperature and pressure differences between the VBL and the plain region CBL, every half hour. (top) Narrow valley; (bottom) wide valley. In all frames, profiles evolve in time from an unperturbed state where the atmosphere has the same vertical structure both in the valley and in the plain (light gray) to a final condition where the contrast between VBL and CBL is greatest (black).

the high-altitude thermal structure of the atmosphere were favorable. In particular, the presence of an elevated warm layer in the VBL would be sufficient to cause a down-valley pressure gradient (and consequently an upvalley wind) even if the surface temperature in the valley were lower than on the plain.

Conversely, a down-valley pressure gradient force at high altitudes would arise as a consequence of elevated potential cooling above the valley: a similar situation occurs for example in our narrow valley case, where turbulent entrainment around the mountaintop plumes produces a cooling effect at an altitude at which the free atmosphere above flat terrain is unperturbed. The atmosphere would respond to this thermal imbalance with an upper-level valley-to-plain circulation.

This perspective offers an alternative interpretation of the valley-plain flow occasionally observed at high altitude above the up-valley breeze (Whiteman 2000).
The antiwind aloft might be driven by a local thermal imbalance, rather than arise as a compensation current required by mass conservation. In fact, it is misleading to suggest that the antiwind aloft does arise as a consequence of mass continuity, because the atmosphere within and above a valley is not a closed system. If it were caused merely by mass compensation, then the antiwind would be a necessary counterpart of all nearsurface up-valley circulations, which is not. Actually, antiwinds are rather rarely observed (Whiteman 2000): recognizing that they are driven by a weak thermally induced pressure gradient aloft can justify why the phenomenon is so elusive. In particular, such a wind would not occur if ambient flow above the ridge tops advected downwind the elevated heat input operated by slope flows. This would damp the growth of the VBL and prevent the onset of the weak high-level pressure maximum right above the valley. However, further investigations 

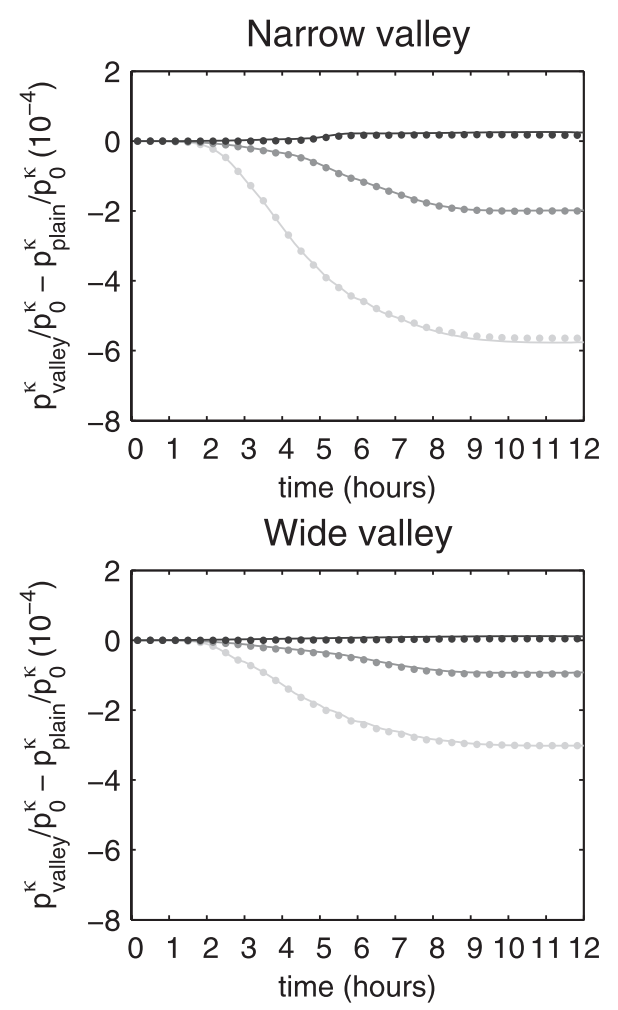

FIG. 7. Nondimensional pressure contrast between valley and plain at altitudes of 1000 (gray), 2000 (dark gray), and $3000 \mathrm{~m}$ (black); $\kappa=R / c_{p}, p_{0}=1000 \mathrm{hPa}$. The continuous line represents the observed difference, while dots indicate the expected contrast based on Eq. (14).

providing evidence from fully $3 \mathrm{D}$ simulations are required to support this hypothesis.

Note that the valley-plain temperature and pressure differences (Fig. 6) reach their maximum intensity about $5 \mathrm{~h}$ from the beginning of simulations, while they remain substantially unaltered later on. This means that the largest fraction of the thermal imbalance between valley and plain builds up before hour 5-that is, mostly during the morning transition phase, when slope winds are typically the dominant flow pattern.

In the valley-plain wind system, the region close to the valley inlet will experience the onset of an up-valley wind component, which may significantly alter the heat transfer processes through the related along-valley cold advection, as clearly shown by Schmidli and Rotunno (2010). Moreover, in a valley with a sloping floor, as most real valleys are, an up-valley breeze would start developing early in the morning all along the valley and thus interact with cross-valley flow, possibly with considerable effects on the heat transfer processes outlined in this study. Nevertheless, even in these dynamically more complex situations, the enhanced BL growth in mountain regions suggests a possible mechanism for the occurrence of both a daytime up-valley flow (as a consequence of high-altitude warming in mountain regions related to the advection operated by slope flows) and of a return flow aloft (from higher-level entrainment near mountaintops and the related local cooling).

\section{Conclusions}

The analysis provided in Serafin and Zardi (2010a) was extended to gain insight into the buildup of the thermal imbalance driving up-valley motions during daytime. The plain and valley environments were studied with separate LES, representing the asymptotic thermal structures that occur far up valley and on the adjacent plain, assuming a horizontal floor at the same level of the plain and an invariant cross-valley topographic profile. A comparison between the two separate environments provides valuable information about the $\mathrm{BL}$ processes occurring in areas far enough from the inlet to be unaffected by the up-valley wind.

Thermal imbalances between the CBL above a plain area (ideally representing the thermal profile above the plain, out of the valley) and the VBL (ideally representing the state of the atmosphere far up valley) arise because of different heat transfer processes. In particular, downward heat advection in the valley core compensating upslope flows is responsible for a "top-down" heating process in valleys, which is not observed above flat topography. Top-down heating results initially from the downward displacement of potentially warm air from the free atmosphere (occurring at midvalley to compensate upslope motion), and later from the heat continuously supplied by stationary thermal plumes on mountaintops (Serafin and Zardi 2010a).

Such an elevated heat source causes the VBL to extend to higher altitudes and to have a larger mean potential temperature than the CBL, under comparable thermal forcing. The heated volume in the VBL and CBL appears to be of equal size and subject to a comparable mean potential temperature increment during daytime. Since the VBL stretches to a higher altitude, its mean potential temperature is initially warmer than that of the CBL. As the VBL progressively gets well mixed, the potentially warm air available at high altitudes is transferred to lower levels, causing a significant increase of the surface potential temperature.

Positive temperature differences between the VBL and the CBL are larger around the level of mountaintops. Small negative temperature differences can be observed at higher altitudes, if the VBL is effective in entraining air from the free atmosphere. The magnitude of the entrainment heat flux in the VBL appears to be enhanced in relatively narrow valleys. 
High-altitude thermal imbalances can cause surface pressure differences between the valley and the plain. The occurrence of a comparatively warm elevated layer in the VBL can then be responsible for a local surface pressure minimum, possibly driving an up-valley breeze. Apparently, the latter can develop even if the surface temperature within the valley is lower than above an adjacent plain, as a thermal contrast at an upper level is sufficient to drive such a flow.

We believe that these findings can cast a new light on the volume-effect concept. In fact, the arguments presented here prove that the geometry of a valley's topography actually does have a decisive impact in determining its heating rate. However, a key point to obtain consistent estimates of the bulk heating rate of a valley is the correct evaluation of the volume of atmosphere affected by the circulation induced by slope flows. Unlike what traditional approaches suggest (e.g., Steinacker 1984; Whiteman 1990), volume-effect evaluations need to consider a control volume that may extend well beyond the mountaintops. An immediate consequence of this conclusion is the recognition that enhanced near-surface heating in valleys is a consequence of the mixing into the VBL of potentially warm air originally lying above the mountaintops.

Furthermore, it has been shown that differences in the vertical profiles of temperature and pressure between the VBL and the CBL reach their maximum amplitude already in the early afternoon. This makes us confident that our conclusions can be of general validity since along-valley circulations, which cannot be accounted for in the present simplified setting, are usually relevant only in the later stages of the diurnal cycle and in a limited region close to the valley inlet.

To make progress toward the understanding of processes occurring in real valleys, further aspects need to be explored. One of them is the effect of a sloping floor, not considered in our analysis. In the presence of a sloping valley floor, buoyancy-induced up-valley motions are expected not just to propagate from the valley inlet after a valley-plain contrast has built up but rather to start simultaneously all along the valley length, whatever the slope angle and the forcing intensity. Indeed, various field measurements showed that up-valley motions can be produced even by slightly tilted valley floors (Whiteman and Zhong 2008; Zhong and Whiteman 2008; de Franceschi et al. 2009) or under very weak thermal forcings (de Franceschi and Zardi 2009). As a consequence, heat transfer processes and their connection to cross- and along-valley flows, as well as their respective time scales, may significantly change under different combinations of topographic features (in particular the cross section width-to-depth ratio and the sidewalls and floor slope angles).
Further aspects deserving investigation are the implications of the breakup of a nocturnal inversion at the bottom of the valley, which may considerably modify the early stages of the VBL development, and those of large-scale motions above the valley crests, potentially causing significant heat advection to or from the valley atmosphere.

Finally, it has to be emphasized that the numerical setup adopted in the present study does not allow assessment of the implications of the along-valley wind. An obvious guess is that it would act to restore the pressure imbalances originated earlier on, as found in threedimensional simulations of the valley-plain wind system (Li and Atkinson 1999; Rampanelli et al. 2004; Schmidli and Rotunno 2010). However, in those studies high spatial resolution and accuracy of the turbulence closure scheme were sacrificed to implement an appropriately comprehensive simulation domain. Fully 3D large-eddy simulations of the coupled valley-plain environment are now feasible, and are expected to extend and provide further support to the results of the present study.

Acknowledgments. The authors acknowledge support from CINECA, the Italian national supercomputing centre, where the computations for this study were performed. D. Zardi is grateful to the University of Trento for granting him a sabbatical leave during the academic year 2009/10.

\section{REFERENCES}

de Franceschi, M., and D. Zardi, 2009: Study of wintertime high pollution episodes during the Brenner-South ALPNAP measurement campaign. Meteor. Atmos. Phys., 103, 237-250.

_ - G. Rampanelli, D. Sguerso, D. Zardi, and P. Zatelli, 2003: Development of a measurement platform on a light airplane and analysis of airborne measurements in the atmospheric boundary layer. Ann. Geophys., 46, 269-283.

_ D. Zardi, M. Tagliazucca, and F. Tampieri, 2009: Analysis of second order moments in the surface layer turbulence in an Alpine valley. Quart. J. Roy. Meteor. Soc., 135, 1750-1765.

De Wekker, S. F., 2002: Structure and morphology of the convective boundary layer in mountainous terrain. Ph.D. thesis, Department of Earth and Ocean Sciences, University of British Columbia, 191 pp.

Egger, J., 1990: Thermally induced flow in valleys with tributaries. Part I: Response to heating. Meteor. Atmos. Phys., 42, 113-125.

Gill, A. E., 1982: Atmosphere-Ocean Dynamics. Academic Press, $662 \mathrm{pp}$.

Li, J.-G., and B. W. Atkinson, 1999: Transition regimes in valley airflows. Bound.-Layer Meteor., 91, 385-411.

Moeng, C.-H., and J. C. Wyngaard, 1984: Statistics of conservative scalars in the convective boundary layer. J. Atmos. Sci., 41, 3161-3169.

Noilhan, J., and S. Planton, 1989: A simple parameterization of land surface processes for meteorological models. Mon. Wea. Rev., 117, 536-549. 
Oke, T. R., 1987: Boundary Layer Climates. 2nd ed. Routledge, 435 pp.

Rampanelli, G., and D. Zardi, 2004: A method to determine the capping inversion of the convective boundary layer. J. Appl. Meteor., 43, 925-933.

_,$\ldots$, and R. Rotunno, 2004: Mechanisms of up-valley winds. J. Atmos. Sci., 61, 3097-3111.

Reuten, C., D. G. Steyn, and S. E. Allen, 2007: Water tank studies of atmospheric boundary layer structure and air pollution transport in upslope flow systems. J. Geophys. Res., 112, D11114, doi:10.1029/2006JD008045.

Rotach, M. W., and D. Zardi, 2007: On the boundary-layer structure over highly complex terrain: Key findings from MAP. Quart. J. Roy. Meteor. Soc., 133, 937-948.

Schmidli, J., and R. Rotunno, 2010: Mechanisms of along-valley winds and heat exchange over mountainous terrain. J. Atmos. Sci., 67, 3033-3047.

_ - and Coauthors, 2011: Intercomparison of mesoscale model simulations of the daytime valley wind system. Mon. Wea. Rev., 139, 1389-1409.

Serafin, S., and D. Zardi, 2010a: Daytime heat transfer processes related to slope flows and turbulent convection in an idealized mountain valley. J. Atmos. Sci., 67, 3739-3756.

— and 2010b: Structure of the atmospheric boundary layer in the vicinity of a developing upslope flow system: A numerical model study. J. Atmos. Sci., 67, 1171-1185.

Steinacker, R., 1984: Area-height distribution of a valley and its relation to the valley wind. Beitr. Phys. Atmos., 57, 64-71.

Stull, R. B., 1988: An Introduction to Boundary Layer Meteorology. Kluwer Academic, 666 pp.

Sullivan, P. P., C.-H. Moeng, B. Stevens, D. H. Lenschow, and S. D. Mayor, 1998: Structure of the entrainment zone capping the convective atmospheric boundary layer. J. Atmos. Sci., 55, 3042-3064.

Vergeiner, I., 1987: An elementary valley wind model. Meteor. Atmos. Phys., 36, 255-263.
— , and E. Dreiseitl, 1987: Valley winds and slope windsObservations and elementary thoughts. Meteor. Atmos. Phys., 36, 264-286.

Wagner, A., 1932: Der tägliche Luftdruck- und Temperaturgang in der freien Atmosphäre und in Gebirgstälern. Gerlands Beitr. Geophys., 37, 315-344.

Wenger, R., 1923: Neue Theorie der Berg- und Talwindes. Meteor. Z., 40, 193-204.

Whiteman, C. D., 1990: Observations of thermally developed wind systems in mountainous terrain. Atmospheric Processes over Complex Terrain, Meteor. Monogr., No. 23, Amer. Meteor. Soc., 5-42.

_ 2000: Mountain Meteorology: Fundamentals and Applications. Oxford University Press, 355 pp.

— , and T. B. McKee, 1982: Breakup of temperature inversions in deep mountain valleys: Part II. Thermodynamic model. J. Appl. Meteor., 21, 290-302.

— and their interactions with valley inversions. Part I: Observations. J. Appl. Meteor. Climatol., 47, 2023-2038.

Xue, M., K. K. Droegemeier, and V. Wong, 2000: The Advanced Regional Prediction System (ARPS): A multi-scale nonhydrostatic atmospheric simulation and prediction model. Part I: Model dynamics and verification. Meteor. Atmos. Phys., 75, 161-193.

- - - , and — 2001: The Advanced Regional Prediction System (ARPS): A multi-scale nonhydrostatic atmospheric simulation and prediction tool. Part II: Model physics and applications. Meteor. Atmos. Phys., 76, 143-165.

Zardi, D., and C. D. Whiteman, 2012: Diurnal mountain wind systems. Mountain Weather Research and Forecasting, F. K. Chow, S. F. J. De Wekker, and B. Snyder, Eds., Springer, in press.

Zhong, S., and C. D. Whiteman, 2008: Downslope flows on a low-angle slope and their interactions with valley inversions. Part I: Numerical modeling. J. Appl. Meteor. Climatol., 47, 2039-2057. 